\title{
Management and follow-up of a patient after bariatric surgery
}

\author{
Cem Kaan Parsak \\ Department of General Surgery, Cukurova University Faculty of Medicine, Adana, Turkey
}

\begin{abstract}
Long-term bariatric follow-up requires team approach and attention to several aspects of care. Active nutritional patient education and clinical management to prevent and detect nutritional deficiencies are recommended for all patients who undergo bariatric surgery. Patients who make dietary and lifestyle changes as an adjunct to their surgical procedure have better nutritional and weight loss outcomes than those who have limited follow-up. This article provides management and follow-up strategies for nutritional and dietary considerations of patients after bariatric surgery.
\end{abstract}

Keywords: Bariatric; bariatric surgery; long-term follow-up; management; nutrition.

\section{Introduction}

Bariatric surgery refers to a variety of surgical procedures whose primary goal is weight loss through malabsorption, restriction, or a combination of the two, depending on the type of procedure performed. Malabsorptive procedures like biliopancreatic diversion (BPD) and biliopancreatic diversion with duodenal switch (BPD/DS) work by bypassing the intestinal lumen where most nutrient absorption occurs. Restrictive procedures [laparoscopic adjustable gastric banding (LAGB), sleeve gastrectomy (SG), and vertical banded gastroplasty (VGB)] primarily limit the volume of food ingested. Roux-en-Y gastric bypass (RYGB) achieves weight loss through a combination of malabsorption and restriction. For management and follow-up of the bariatric patients, the type of surgical procedure they underwent is very important.

Morbid obesity is a lifelong disease. Bariatric surgery is an effective method of weight loss for the treatment of morbid obesity. For the success of surgery, medical physician and surgeon are responsible for the treatment of co-morbidities before the operation and the follow-up after the operation. It is well known that all bariatric procedures affect nutritional intake and some procedures may affect the absorption of macronutrients and/or micronutrients. Therefore patients will be required to stay on lifelong nutritional supplements and have lifelong monitoring of their nutritional status. Several organizations have provided guidelines on the most effective management of individuals having had bariatric surgery ${ }^{[1-4]}$ It is assumed that the goal of such guidelines is to maximize the quality of care these patients receive.

During the follow-up period of weight loss, patients should be observed closely. In the follow-up period, main goals 
are to evaluate nutrition status and weight loss, identify eating disorders, evaluate potential complications of surgery, control preexisting comorbidities, encourage regular exercise, and control the required laboratory values. ${ }^{[4,5]}$ The success of bariatric surgery and weight loss goals are measured by excess body weight (EBW) loss, which is calculated by subtracting current body weight from ideal body weight.

\section{Nutrition Management and Eating Behavior Considerations}

All bariatric surgery patients are at risk for nutrition deficiencies. Nutrition is the most important aspect of follow-up to increase weight loss and prevent weight regain. Dietary management is based on the amount of time since the surgery was performed and may be divided into early (up to postoperative six months) and long-term maintenance periods.

Many patients have maladaptive eating behaviors, nutritional deficiencies or in adequacies and these issues cannot be corrected just with bariatric surgery. Nutritional management of these patients requires both behavioral modification of eating habits and modifications of the content and quality of food consumed. In case of lack of resolution, patients' chances for success after surgery decreases. ${ }^{[2]}$ Compliance with dietary recommendations is very important as the patient transitions from a period of rapid weight loss during the first year to a period of longerterm weight stabilization and possible weight regain. ${ }^{[6]}$

Patients may report common dietary related complaints or adverse gastro-intestinal symptoms after bariatric surgery. The different mechanisms of action of each surgical procedure may also have a distinct influence on eating behaviors. ${ }^{[7,8]}$ After SG and RYGB, the reduced gastric volume combined with hormonal changes, taste changes and, in the case of SG, increased gastric emptying, influence eating style of patients. Dysfunctional eating behavior may result in discomfort, regurgitation and dumping syndrome. ${ }^{[8-10]}$ Therefore, by adding simple changes to a patient's eating and drinking style, adverse symptoms can be minimized. As a result, these may help patients to adjust and establish new eating and drinking behaviors more easily.

During early postoperative period, primary goals are to maintain adequate hydration, provide adequate fluid, nutrients and protein to support healing and minimize loss of lean muscle mass and progressively return to "normal" food. ${ }^{[1,2]}$ Most patients are discharged from the hospital on a full liquid diet, therefore, patients should be taught to keep monitoring their hydration and urine output. Approximately two-three weeks after surgery, diet is gradually changed to soft, solid foods. The average calorie intake ranges from 400 to $1200 \mathrm{kcal} / \mathrm{d}$ for the first month. ${ }^{[2]}$

There are post-operative recommendations for specific nutrients and eating patterns. Guidelines suggest that patients should eat slowly and chew food thoroughly to avoid dumping syndrome. ${ }^{[2,1]}$ Moreover, it is recommended that patients do not consume food and beverages at the same time and ingest liquids within 30 minutes of a meal.

${ }^{[2,11]}$ For patients suffering from chronic vomiting, prokinetic therapy and proton-pump inhibitors should be considered. Patients, who underwent SG, LAGB or RYGB, benefit from a well-planned dietary advancement. Therefore, educating patients based on the type of surgical procedure they underwent is very important. Patients should know the importance of self-monitoringby means of keeping daily food records.

Protein intake is important since deficits in protein intake can lead to fat storage and breakdown of lean muscle mass, which may adversely affect weight loss efforts. [1] Protein recommendations should be based on the type of surgical procedure. Most patients are able to consume $0.8-1 \mathrm{~g}$ of protein $/ \mathrm{kg}$ of ideal body weight. For restrictive procedures (i.e., LAGB or RYGB), protein requirements are between 60 and $120 \mathrm{~g} /$ day. Patients who have undergone a malabsorptive procedure (BPD or BPD/DS) should consume between 80 and $120 \mathrm{~g} /$ day ${ }^{[5]}$ Carbohydrates (i.e., bread, rice, and pasta) should be avoided until the patient is consuming 60 grams of protein per day plus fruits and vegetables. ${ }^{[2,6,8]}$ Another concern is fluid status. Appropriatehydration is necessary for patients during the period of rapid weight loss. Patients should consume approximately 1.5 liters of fluid each day to maintain adequate hydration. ${ }^{[2]}$ However, if food intolerance develops, patients may prefer a more vegetarian-based diet. Fresh fruits and vegetables are usually well tolerated by patients.

\section{Vitamin, Mineral and Trace Element Monitoring and Supplementation}

Nutrient deficiencies vary depending on the type of surgical procedure performed. Pure gastric restrictive procedures are not associated with alterations in intestinal continuity and do not alter normal digestive physiology. As a result, selective nutritional deficiencies are uncommon. 
Therefore, evaluation of deficiencies are recommended after bariatric surgeries to detect subclinical nutritional deficiencies and prevent development of frank deficiencies. ${ }^{[12,13]}$ Nutrient deficiencies may be identified through laboratory testing or by presentation of clinical symptoms.

Malabsorptive procedures can be associated with micronutrient and macronutrient deficiencies and require lifelong supplementation and monitoring of laboratory data by a physiciancaring for a post-surgical bariatric patient, who has, at a minimum, basic understanding of the nutrient deficiencies and other nutritional concerns of his/ her patient. ${ }^{[12-14]}$ Baseline data should be obtained before bariatric surgery to allow correction of deficiencies and to provide comparison values. Anatomic changes created by malabsorptive surgery increase the risk for various vitamin and mineral deficiencies, commonly within the first year after surgery. ${ }^{[4,12,13]}$ Best practice guidelines published recently recommend a daily multivitamin and calcium supplementation with added vitamin D for all weight-loss surgery patients. ${ }^{[14]}$ Calcium deficiency occurs asprimary absorptionsites (duodenum and proximal jejunum) maybe bypassed. Decreased absorption of calcium may cause osteoporosis and metabolic bone disease. Recommended doses of elemental calcium citrate after bariatric surgery range from 1200-2000 mg daily and these usually contain vitamin $\mathrm{D}$, as well. ${ }^{[3-5]}$ Calcium citrate preparations are preferred since it is better absorbed in the absence of gastric acid production. ${ }^{[4,15]}$ Calcium and vitamin D can also be given as separate supplements. Calcium carbonate preparations are available in chewable forms and are better tolerated shortly after surgery. However, patients should be advised to take calcium carbonate preparations with meals in order to enhance intestinal absorption or increase the dosage to $2000 \mathrm{mg} / \mathrm{d}$.

Vitamin B12 deficiencies can occur after bariatric surgery procedures that bypass the lower stomach. Impairment of vitamin B12 absorption after RYGB results from decreased digestion of the protein-bound cobalamins and impaired formation of intrinsic factor-vitamin B12 complexes required for absorption. ${ }^{[16,17]}$ Vitamin B12 supplementation within 6 months postoperatively is recommended. Oral crystalline vitamin B12 at a dose of at least $350 \mathrm{mg} / \mathrm{d}$ has been shown to maintain normal plasma vitamin B12 levels. ${ }^{[18]}$ Regardless of the preparation, multivitamin supplements providing $400 \mathrm{mg} / \mathrm{d}$ folate can effectively prevent the development of folate deficiency after RYGB. ${ }^{[4,19]}$ The intake of folic acid from the diet and routine multivitamins is generally sufficient to prevent folic acid deficiency.

Malabsorptive procedures cause food to bypass parts of the duodenum and jejunum, where most iron and calcium are absorbed. It was found that vitamin D and calcium deficiency increase significantly with the length of the Roux limb; consequently, this is another factor that should be considered when prescribing supplements to these patients. ${ }^{[5]}$ Iron deficiency has been reported to occur in up to $50 \%$ of the patients after RYGB, most frequently in women with menorrhagia. ${ }^{[20]}$ Since oral iron supplementation is associated with poor absorption and adverse gastrointestinal effects and intramuscular injections are painful, intermittent intravenous iron infusion may be required during treatment. ${ }^{[4,20]}$

Patients who have had a duodenal switch or long limbed gastric bypass are at the greatest risk of malabsorption and steatorrhoea. Consequently, these patients may have deficiencies in fat-soluble vitamins and zinc, which typically present as an eczematous rash. ${ }^{[19,21]}$ Deficiency is usually prevented if the patient takes a daily multivitamin.

Thiamine deficiency can occur as a result of bypass of the jejunum, where thiamine is primarily absorbed or as a result of impaired nutritional intake from recurrent emesis. ${ }^{[5,22]}$ Severe thiamine deficiency most commonly occurs in patients who develop severe, intractable vomiting after bariatric surgery, usually due to a mechanical problem like stenosis. Clinical presentations include acute Wernicke encephalopathy (nystagmus, ophthalmoplegia, ataxia, and confusion), lower limb hypotonia, seizures, polyneuropathy, unsteady gait and ataxia, and hearing loss. These clinical findings of thiamine deficiency have been reported as soon as $1-3$ months after surgery. ${ }^{[5,23]} \mathrm{Se}$ vere deficiency is associated with beriberi. Diagnosis can be made by measuring erythrocyte transketolase activity, blood thiamine concentration, or transketolase urinary thiamine excretion. Patients should receive daily B-complex supplements to prevent deficiency. ${ }^{[4,5]}$

The multivitamin-mineral preparations should have the recommended daily requirements for vitamins and minerals. Initially, one to two tablets of a chewable preparation is advised because they are better tolerated after malabsorptive procedures. However, nonchewable preparations or products with increased amounts of folic acid and iron, like prenatal vitamins, can be used (Table 1). 
Planned lifelong testing for nutrient markers is recommended following all procedures. In the first year, baseline tests should be repeated every 6 months after RYGB and SG, on the anniversary for AGB, and then annually thereafter for all procedures (Table 2). ${ }^{[1-4]}$

\section{Common Complaints and Solutions for Gastrointestinal Symptoms}

Vomiting is most common during the first few postoperative months when patients are adapting to a small gastric pouch. ${ }^{[4]}$ Recurrent vomiting needs to be addressed urgently, particularly in the first 8 weeks after RYGB and SG surgery as it may lead to thiamine depletion and dehydration. Vomiting could be a result of stenosis/anastomic stricture following SG or RYGB, generally occurring around 8 weeks post-operatively. Nausea and vomiting are caused by overeating or by eating too quickly. To prevent this, patients should eat slowly, chew foods very well, keep to recommended portion sizes and stop eating as soon as they feel full. ${ }^{[4,23,24]}$ Patients should be aware that eating and drinking together are incompatible, espe- cially following SG and RYGB. ${ }^{[10]}$

Constipation occurs because the intake of food and fiber is reduced following surgery. Patient may confuse reduced frequency/volume of bowel output due to reduced intake with constipation. Prevention tips include encouraging adequate fluid (1000-1500 mL/day), high fiber intake (25-30 g/day),exercise and taking fiber supplement. ${ }^{[4,5]}$

Dumping syndrome is more common after RYGB and SG, mostly caused by food emptying too quickly from the stomach. Symptoms include diarrhea, nausea, cold sweats, and light-headedness. In order to prevent dumping syndrome, patients should avoid consuming refined sugars and high-fat foods and wait 30 minutes after meals before fluid intake. ${ }^{[1,25]}$

Diarrhea may be a transient post-operative event and a clinical finding of dumping syndrome. Soluble fiber in some circumstances can be added to the diet. ${ }^{[5]}$

Gallstones occur in approximately 20\% of RYGB patients. Patients with symptomatic gallstones may undergo chole-

\section{Table 1. Guidelines for supplement administration ${ }^{[5]}$}

- One chewable vitamin/mineral tablet should be taken at breakfast and at dinner for 6 months after surgery (after 6 months, change to tablet)

- Calcium citrate should be taken at mid-morning and midafternoon (500-600 mg twice daily)

- $\mathrm{AB}$-complex vitamin with at least $10 \mathrm{mg}$ of thiamin should be taken

- Vitamin B1 sublingual (500 mg daily, $1200 \mathrm{mg}$ bi weekly or $500 \mathrm{mg}$ weekly) or a monthly injection of $1 \mathrm{~mL}$

- If extra iron is needed, it should be taken with vitamin C (allow $2 \mathrm{~h}$ or longer between iron and calcium supplements to avoid interference with absorption)

Table 2. Recommended postoperative nutritional monitoring ${ }^{\star[2]}$

\begin{tabular}{|c|c|c|c|c|}
\hline Recommendation & AGB & VSG & RYGB & BPD-DS \\
\hline Bone density (DXA) at 2 years & Yes & Yes & Yes & Yes \\
\hline 24 hour urinary calcium excretion at 6 months and annually & Yes & Yes & Yes & Yes \\
\hline $\begin{array}{l}\text { Vitamin B12 annually (methylmalonic acid and homocysteine optional) } \\
\text { then every 3-6 months if supplemented }\end{array}$ & Yes & Yes & Yes & Yes \\
\hline $\begin{array}{l}\text { Folic acid (red blood cell folic acid optional), iron studies, vitamin D, } \\
\text { intact parathyroid hormone }\end{array}$ & No & No & Yes & Yes \\
\hline Vitamin A initially and every 6-12 months thereafter & No & No & Optional & Yes \\
\hline Copper, zinc, and selenium evaluation with specific findings & No & No & Yes & Yes \\
\hline Thiamine evaluation with specifi c findings & Yes & Yes & Yes & Yes \\
\hline
\end{tabular}

*AGB: Adjustable gastric banding; BPD-DS: Biliopancreatic diversion with duodenal switch; DXA: Dual energy X-ray absorptiometry; RYGB: Roux-en-Y gastric bypass; VSG: Vertical sleeve gastrectomy. 
cystectomy. Ulcers of the proximal jejunum may develop after surgery. Smoking and nonsteroidal anti-inflammatory agent (NSAID) use exacerbate these ulcers and must be stopped. Proton-pump inhibitor therapy may also be beneficial. NSAIDs should be discontinued after surgery due to an increased risk of bleeding and acetaminophen should be preferred instead. ${ }^{[4,26]}$

\section{Medical Considerations}

Contraception counseling is recommended for women, who may have regained fertility as a result of weight loss. Pregnancy should be delayed for 12 to 18 months after surgery. ${ }^{[27]}$

Temporary hair loss is caused by rapid weight loss and/or lack of protein or vitamins/minerals in the diet. ${ }^{[5]}$ To prevent this, patients should consume the amount of protein recommended and take vitamins/minerals as suggested.

\section{Management of Diabetes Mellitus and Lipids}

After RYGB or BPD/DS/ GS, insulin-treated patients experience a significant decrease in insulin requirements; the majority of patients can discontinue insulin therapy by 6 week after surgery and some may even be able to discontinue insulin before hospital discharge. ${ }^{[28]}$

It is recommended that postoperative glycemic control should consist achieving glycated hemoglobin (HbA1c) of $7 \%$ or less, with fasting blood glucose no greater than 110 $\mathrm{mg} / \mathrm{dL}$ and postprandial glucose no greater than $180 \mathrm{mg} /$ dL. ${ }^{[4]}$ Improvements in hyperglycemia are observed almost immediately after RYGB, partly due to increased release of GLP-1 and possibly other incretins. ${ }^{[29]}$ It was suggested that patients who present with post prandial symptoms of hypoglycemia, particularly neuroglycopenic symptoms, should undergo furthere valuation for the possibility of insulin-mediated hypoglycemia. ${ }^{[4]}$

Obese patients will have abnormal liver function tests and these changes are most commonly associated with fatty liver disease or nonalcoholic fatty liver disease. ${ }^{[30]} \mathrm{Ab}$ normal transaminases should be followed at appropriate intervals until they fall into the normal range or remain constant. Triglyceride and LDL -cholesterol decrease and high-density lipoprotein-cholesterol increases after AGB, RYGB, BPD, or BPD/DS surgery. ${ }^{[4,31]}$

\section{Bone Health and Gout}

It was recommended that patients who have undergone malabsorbtive (i.e. RYGB, GS, and BPD) surgical procedures should have vitamin D, calcium, phosphorus, PTH, and alkaline phosphatase levels followed every 6 months and have a dual-energy X-ray absorptiometry for bone density performed yearly until stable. ${ }^{[2,4]}$ It has been suggested that patients with frequent attacks of gout should have prophylactic therapy to lessen the chance of acute gout postoperatively as they lose weight. ${ }^{[2,4]}$

\section{Psychosocial Risks}

Data from observational studies suggest that some bariatric procedures introduce a greater long term risk of substance misuse disorders, suicide, and nutritional deficiencies. ${ }^{[2,4]}$ Pharmaco kinetic studies indicate that the gastrointestinal anatomy after RYGB and SG leads to more rapid absorption of alcohol and marked increases in blood alcohol concentrations per dose. This may in advertently increase the frequency of physiological binges and subsequent alcohol misuse disorder. ${ }^{[4,23,32]}$ Pre-operative identification of psychological risk factors associated with lower postoperative compliance, inadequate weight loss, alcohol or drug dependencies, eating pathologies and others should lead to post-operative interventions through implementing a selfmonitoring strategy in higher-risk patients.

\section{Expected Rates of Weight Loss and Weight Regain}

Many patients often have unrealistic expectations for both the rate and total weight loss expected after restrictive surgery. Although the rate of weight loss varies between surgeries and individuals, up to $4 \mathrm{~kg}$ weight loss per month is a reasonable expectation. ${ }^{[33]}$ Following

\section{Table 3. Causes and prevention of weight regain ${ }^{[3]}$}

Causes

Noncompliance with dietary and lifestyle recommendations

Physiological factors (variations in response to surgery)

Surgical failure

Prevention

Optimizing patient selection criteria

Realistic preoperative expectations

Consideration of benefits of bypass vs. restrictive procedures

Adherence to scheduled visits 
guidelines about food choices and physical exercises will promote adequate weight loss and provide maintenance. Unfortunately, most patients will not be able to attain ideal body weight so the goal is to maintain $70 \%$ EBW loss for RGBY and 50\% for AGB. Pure restrictive operations are more commonly associated with weight regain and weight loss failure than other techniques with a malabsorptive component.

More than half of the patients may regain $20-50 \%$ of weight lost in 10 years. On the other hand, loss of patients to follow-up at late stages may underestimate the true prevalence of weight regain. Weight regain is related to noncompliance with dietary and lifestyle instructions although differences in physiological responses and occasionally surgical failure can be the cause (Table 3). It has been recommend that treatment of weight regain postoperatively should include a multidisciplinary approach to medical weight loss, including diet instruction, increased activity, behavior modification, and pharmacological therapy. ${ }^{[4]}$

\section{Conclusion}

Bariatric surgery is a reasonably safe and effective method of weight loss for the treatment of morbid obesity. Main factors contributing to successful weight loss after bariatric surgery are the patient's ability to make lifestyle changes including healthy well-balanced diet, taking recommended vitamin supplementation, and exercising regularly and maintaining those changes for years after surgery. All bariatric procedures affect nutritional intake and some procedures may affect the absorption of macronutrients and/or micronutrients. Patients will be required to stay on lifelong nutritional supplements and have lifelong monitoring of their nutritional status.

\section{References}

1. Allied Health Sciences Section Ad Hoc Nutrition Committee, Aills L, Blankenship J, Buffington C, Furtado M, Parrott J. ASMBS Allied Health Nutritional Guidelines for the Surgical Weight Loss Patient. Surg Obes Relat Dis 2008;4(5 Suppl):S73-108.

2. Mechanick JI, Youdim A, Jones DB, Garvey WT, Hurley DL, McMahon MM, et al. Clinical practice guidelines for the perioperative nutritional, metabolic, and nonsurgical support of the bariatric surgery patient--2013 update: cosponsored by American Association of Clinical Endocrinologists, The Obesity Society, and American Society for Metabolic \& Bariatric Surgery. Obesity (Silver Spring) 2013;21 Suppl 1:S1-27.

3. Fried M, Yumuk V, Oppert JM, Scopinaro N, Torres AJ, Weiner
R, et al; European Association for the Study of Obesity; International Federation for the Surgery of Obesity - European Chapter. Interdisciplinary European Guidelines on metabolic and bariatric surgery. Obes Facts 2013;6:449-68.

4. Heber D, Greenway FL, Kaplan LM, Livingston E, Salvador J, Still C; Endocrine Society. Endocrine and nutritional management of the post-bariatric surgery patient: an Endocrine Society Clinical Practice Guideline. J Clin Endocrinol Metab 2010;95:4823-43.

5. Richardson WS, Plaisance AM, Periou L, Buquoi J, Tillery D. Long-term Management of Patients After Weight Loss Surgery. Ochsner J 2009;9:154-9.

6. Thomas JR, Gizis F, Marcus E. Food selections of Roux-en-Y gastric bypass patients up to 2.5 years postsurgery. $\mathrm{J} \mathrm{Am}$ Diet Assoc 2010;110:608-12.

7. Overs SE, Freeman RA, Zarshenas $\mathrm{N}$, Walton $\mathrm{KL}$, Jorgensen JO. Food tolerance and gastrointestinal quality of life following three bariatric procedures: adjustable gastric banding, Roux-en-Y gastric bypass, and sleeve gastrectomy. Obes Surg 2012;22:536-43.

8. Schweiger C, Weiss R, Keidar A. Effect of different bariatric operations on food tolerance and quality of eating. Obes Surg 2010;20:1393-9.

9. Melissas J, Daskalakis M, Koukouraki S, Askoxylakis I, Metaxari M, Dimitriadis E, et al. Sleeve gastrectomy-a "food limiting" operation. Obes Surg 2008;18:1251-6.

10. Tzovaras G, Papamargaritis D, Sioka E, Zachari E, Baloyiannis I, Zacharoulis D, et al. Symptoms suggestive of dumping syndrome after provocation in patients after laparoscopic sleeve gastrectomy. Obes Surg 2012;22:23-8.

11. Laville $M$, Romon $M$, Chavrier G, Guy-Grand B, Krempf $M$, Chevallier JM, et al. Recommendations regarding obesity surgery. Obes Surg 2005;15:1476-80.

12. Ernst B, Thurnheer M, Schmid SM, Schultes B. Evidence for the necessity to systematically assess micronutrient status prior to bariatric surgery. Obes Surg 2009;19:66-73.

13. Xanthakos SA, Inge TH. Nutritional consequences of bariatric surgery. Curr Opin Clin Nutr Metab Care 2006;9:489-96.

14. Shikora SA, Kim JJ, Tarnoff ME. Nutrition and gastrointestinal complications of bariatric surgery. Nutr Clin Pract 2007;22:29-40.

15. Goode LR, Brolin RE, Chowdhury HA, Shapses SA. Bone and gastric bypass surgery: effects of dietary calcium and vitamin D. Obes Res 2004;12:40-7.

16. Clements $R H$, Katasani VG, Palepu R, Leeth RR, Leath TD, Roy $\mathrm{BP}$, et al. Incidence of vitamin deficiency after laparoscopic Roux-en-Y gastric bypass in a university hospital setting. Am Surg 2006;72:1196-202.

17. Behrns KE, Smith CD, Sarr MG. Prospective evaluation of gastric acid secretion and cobalamin absorption following gastric bypass for clinically severe obesity. Dig Dis Sci 1994;39:315-20.

18. Rhode BM, Tamin H, Gilfix BM, Sampalis JS, Nohr C, MacLean LD. Treatment of Vitamin B12 Deficiency after Gastric Surgery for Severe Obesity. Obes Surg 1995;5:154-8. 
19. Skroubis G, Sakellaropoulos G, Pouggouras K, Mead N, Nikiforidis G, Kalfarentzos F. Comparison of nutritional deficiencies after Roux-en-Y gastric bypass and after biliopancreatic diversion with Roux-en-Y gastric bypass. Obes Surg 2002;12:551-8.

20. Saltzman E, Anderson W, Apovian CM, Boulton H, Chamberlain A, Cullum-Dugan D, et al. Criteria for patient selection and multidisciplinary evaluation and treatment of the weight loss surgery patient. Obes Res 2005;13:234-43.

21. Stocker DJ. Management of the bariatric surgery patient. Endocrinol Metab Clin North Am 2003;32:437-57.

22. Davies DJ, Baxter JM, Baxter JN. Nutritional deficiencies after bariatric surgery. Obes Surg 2007;17:1150-8.

23. Chaves LC, Faintuch J, Kahwage S, Alencar Fde A. A cluster of polyneuropathy and Wernicke-Korsakoff syndrome in a bariatric unit. Obes Surg 2002;12:328-34.

24. Collene AL, Hertzler S. Metabolic outcomes of gastric bypass. Nutr Clin Pract 2003;18:136-40.

25. Carvajal SH, Mulvihill SJ. Postgastrectomy syndromes: dumping and diarrhea. Gastroenterol Clin North Am 1994;23:261-79.

26. O'Kane M, Pinkney J, Aasheim E, Barth J, Batterham R, Welbourn R. GP Guidance: Management of nutrition following bariatric surgery August 2014. British Obesity and Metabolic
Surgery Society. Available at: http://www.bomss.org.uk/nutritional-guidelines/. Accessed May 24, 2017.

27. Maggard MA, Yermilov I, Li Z, Maglione M, Newberry S, Suttorp $\mathrm{M}$, et al. Pregnancy and fertility following bariatric surgery: a systematic review. JAMA 2008;300:2286-96.

28. Rubino F, Gagner M, Gentileschi P, Kini S, Fukuyama S, Feng $J$, et al. The early effect of the Roux-en- $Y$ gastric bypass on hormones involved in body weight regulation and glucose metabolism. Ann Surg 2004;240:236-42.

29. Cummings DE, Overduin J, Foster-Schubert KE, Carlson MJ. Role of the bypassed proximal intestine in the anti-diabetic effects of bariatric surgery. Surg Obes Relat Dis 2007;3:10915.

30. Clark JM, Diehl AM. Nonalcoholic fatty liver disease: an underrecognized cause of cryptogenic cirrhosis. JAMA 2003;289:3000-4.

31. Sjöström CD, Lissner L, Wedel H, Sjöström L. Reduction in incidence of diabetes, hypertension and lipid disturbances after intentional weight loss induced by bariatric surgery: the SOS Intervention Study. Obes Res 1999;7:477-84.

32. Arterburn DE, Courcoulas AP. Bariatric surgery for obesity and metabolic conditions in adults. BMJ 2014;349:g3961.

33. Ames GE, Patel RH, Ames SC, Lynch SA. Weight loss surgery: patients who regain. Obes Weight Manag 2009;5:154-61. 